\title{
NEUROSCIENCE
}

\section{Delighting in dopamine}

The genetically encoded sensor dLight1 reports dopamine release in culture, slices and behaving mice.

$\mathrm{D}$ opamine is an important neuromodulator with roles in learning and decision making. Methods to measure dopamine levels in the brain are hampered by low spatial and/or temporal resolution. Examples include the genetically encoded iTango system, which depends on transcription and therefore has low temporal resolution, and CNiFERs, which are cells that are implanted into the tissue of interest and serve as dopamine sensors.

Lin Tian from the University of California, Davis, and her collaborators have developed the genetically encoded indicator dLight1, which enables monitoring of dopamine release at high spatial and temporal resolution. The team replaced a part of the human dopamine D1 receptor with circularly permuted GFP and created variants that differed in their affinity for dopamine and in their dynamic range. Binding of dopamine to these dLight 1 sensors results in a conformational change that causes an increase in fluorescence.

In acute slice preparations of tissues subjected to virus-mediated expression of dLight1, the sensor faithfully reported dopamine release after an electrical stimulus or pharmacological treatment. Furthermore, the researchers monitored dopamine levels in head-fixed mice expressing dLight 1 in the dorsal striatum. Using two-photon microscopy, they found that dopamine levels increased when the mice started running on a treadmill.

Furthermore, Tian and her colleagues recorded optogenetically induced dopamine release in freely moving mice by fiber photometry. dLight1 can also sense endogenous levels of dopamine, as the team showed in a behavioral task involving Pavlovian conditioning and in a visuomotor association task.
dLight 1 joins the company of sensors such as, for example, GCaMP variants and iGluSnFR that have been successfully used to monitor neuronal activity. Tools that report on other neurotransmitters, neuromodulators or second messengers would be helpful in assessing neuronal communication in more detail. Tian and her team have made the first steps toward this goal by applying the same design strategies that worked for dLight1 to other molecules such as serotonin and melatonin.

Nina Vogt

Published online: 31 July 2018

https://doi.org/10.1038/s41592-018-0099-7

Research papers

Patriarchi, T. et al. Ultrafast neuronal imaging of dopamine dynamics with designed genetically encoded sensors. Science 360, 6396 (2018).

\section{R8PR COMMUNICATIONS PHYSICS}

\section{NOW PUBLISHING CONTENT}

\section{A new open access journal for the physical sciences from Nature Research}

Communications Physics publishes high-quality primary research articles, reviews and commentary in all areas of the physical sciences. Papers published in the journal represent significant advances that bring new insight to a specialized area of research.

All papers are handled by experienced in-house professional editors supported by an expert Editorial board.

\section{Submit your research today and benefit from:}

- Thorough peer review

- Fast decision process

- High Nature editorial standards
- High visibility

- $\quad$ CC-BY open access as standard

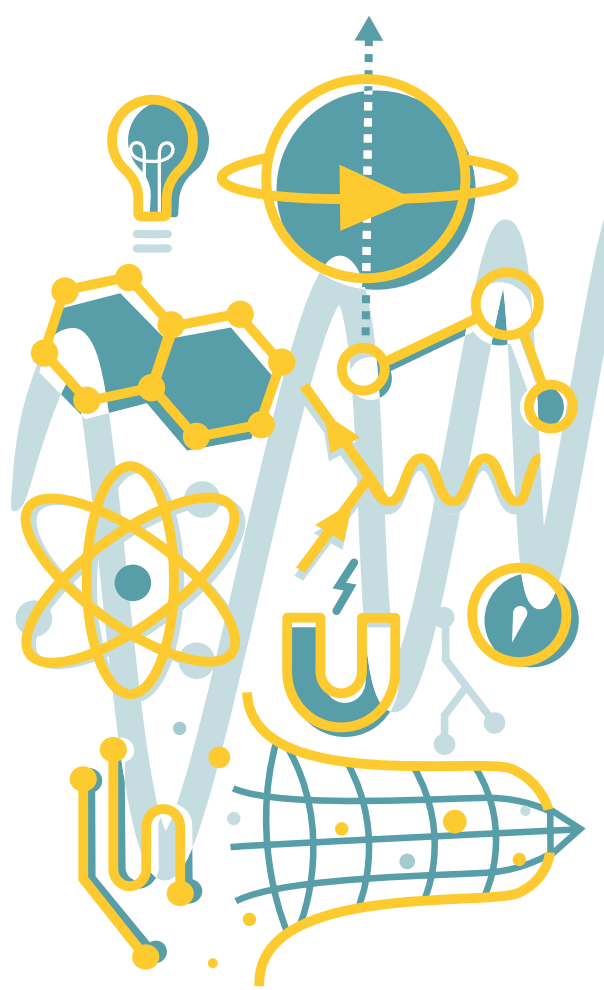

